\title{
The Glucocorticoid Dexamethasone Inhibits U937 Cell Adhesion and Neutrophil Release via RhoA/ROCK1-Dependent and Independent Pathways
}

\author{
Dong Liu ${ }^{a}$ Renping Xionga Xingyun Chen Ping Li Yalei Ning Yan Peng \\ Yan Zhao Nan Yang Yuanguo Zhou
}

State Key Laboratory of Trauma, Burns and Combined Injury, Molecular Biology Center, Research Institute of Field Surgery, Daping Hospital, Third Military Medical University, Chongqing, China; aBoth authors contributed equally to this work

\section{Key Words}

Dexamethasone $\cdot$ Non-genomic effects $\bullet$ ROCK1

\begin{abstract}
Aims: The aim of the present study was to investigate the role of the Ras homolog family member A (RhoA)/Rho-associated coiled-coil-containing protein kinase 1 (ROCK1) signaling pathway in the inhibition of inflammatory responses by the glucocorticoid dexamethasone (Dex). Methods: The inhibitory effects of Dex and Rho-kinase inhibitor fasudil (Fas) on phorbol ester-induced release of $\mathrm{O}_{2}{ }^{-}$and MPO from neutrophils and on U937 mononuclear cell adhesion were examined along with the expression and activity levels of RhoA and ROCK1. Results: High doses of Dex rapidly inhibited the release of $\mathrm{O}_{2}^{-}$and myeloperoxidase (MPO) from neutrophils and the adhesion of U937 cells, while Fas was only found to inhibit U937 cell adhesion. Additionally, Dex suppressed ROCK1 activity. However, Dex had no effects on ROCK1 or RhoA expression levels or on RhoA activity. Neither the glucocorticoid receptor antagonist mifepristone (RU-486) nor the protein synthesis inhibitor cycloheximide (CHX) was able to suppress the effects of Dex $(p>0.05)$. Conclusions: The present findings indicate that Dex suppressed neutrophil release through ROCK1-independent mechanisms and inhibited the adhesion of U937 mononuclear cells through ROCK1-dependent non-genomic mechanisms that did not involve RhoA.
\end{abstract}




\section{Introduction}

Glucocorticoids are known to have a wide range of clinical applications. For example, high-dose glucocorticoid pulse therapy is often used to treat acute inflammation and inflammatory tissue damage, including sepsis and acute lung injury (ALI) [1, 2]. Glucocorticoid pulse therapy is also widely applied for the emergency treatment of asthma and anaphylactic shock. The anti-inflammatory effects of glucocorticoids are generally believed to be mediated by genomic mechanisms, including the inhibition of nuclear factor kappa B (NF$\mathrm{kB}$ ) expression; the downregulation of inflammatory cytokines, such as interleukin-1 beta (IL-1 $\beta$ ) and tumor necrosis factor alpha (TNF- $\alpha$ ), and of adhesion molecules, such as intercellular adhesion molecule 1 (ICAM-1) and vascular cell adhesion molecule 1 (VCAM-1); and positive regulation of the synthesis of anti-inflammatory factor interleukin-10 (IL-10) [3]. However, these genomic mechanisms fail to explain the following effects associated with glucocorticoids: the rapid inhibition of granulocyte adhesion to vascular endothelium, the suppression of granule content release and the rapid therapeutic effects of glucocorticoids on asthma and type I allergy [4]. Although increasing numbers of researchers have shifted their attention to other potential pathways of glucocorticoid action, namely, non-genomic effects, it remains difficult to distinguish between genomic and non-genomic effects. At present, the genomic effects of glucocorticoids are distinguished from their non-genomic effects by the involvement of glucocorticoids in the direct regulation of gene transcription and protein synthesis. The signaling pathways underlying the non-genomic effects of glucocorticoids are poorly understood.

Rho-associated coiled-coil-containing protein kinase (ROCK) is the major downstream effector of Ras homolog family member A (RhoA). The two ROCK subtypes, ROCK1 (ROCK $\beta$ ) and ROCK2 (ROCK $\alpha)$, are mainly involved in stress fiber assembly, cytoskeleton remodeling and focal adhesion complex formation. Previous studies have shown that downregulation of ROCK1 expression effectively reduces the adhesion of inflammatory cells to vascular endothelium [5, 6]. However, the link between ROCK1 and granulocyte release remains largely unknown; it is also unclear whether glucocorticoids exert rapid anti-inflammatory effects through the RhoA/ROCK1 signaling pathway.

The present study utilized rat neutrophils and the mononuclear cell line U937. The glucocorticoid dexamethasone (Dex) and the ROCK-specific inhibitor fasudil (Fas) were combined with blockers of genomic effects, including the glucocorticoid receptor antagonist mifepristone (RU-486 or M) and protein synthesis inhibitors, to investigate the role of the RhoA/ROCK1 signaling pathway in the glucocorticoid-induced rapid inhibition of neutrophil release and mononuclear cell adhesion.

\section{Materials and Methods}

Materials and equipment

Twelve 4- to 6-week old male and female Sprague Dawley (SD) rats weighing approximately $200 \mathrm{~g}$ were obtained from the Experimental Animal Center at the Research Institute of Field Surgery, Daping Hospital, Third Military Medical University. The human mononuclear macrophage cell line U937 and human umbilical vein endothelial cells (HUVEC) were cryogenically preserved in liquid nitrogen at the Molecular Biology Center of the Research Institute of Field Surgery, Daping Hospital, Third Military Medical University. Phorbol 12-myristate 13-acetate (PMA), Dex, RU-486 and cycloheximide (CHX or C) were purchased from Sigma-Aldrich, and Fas was purchased from Asahi Kasei Pharma Corporation. Cell Counting Kit-8 (CCK-8) and the superoxide detection assay kit were purchased from Beyotime Institute of Biotechnology, and the myeloperoxidase (MPO) activity assay kit was purchased from Nanjing Jiancheng Bioengineering Institute.

Methods

Cell isolation and culture. Rat peripheral blood neutrophils were isolated using rat neutrophil isolation solution (TBD Science, China) according to the manufacturer's instructions. U937 and HUVEC cells were 
cultured in Roswell Park Memorial Institute 1640 (RPMI1640) medium containing 10\% fetal bovine serum (FBS) and Dulbecco's Modified Eagle Medium (DMEM) supplemented with 10\% FBS, respectively. The cells were incubated at $37^{\circ} \mathrm{C}$ in a humidified incubator with $5 \% \mathrm{CO}_{2}$.

Examination of the release of superoxide anion radical $\left(\mathrm{O}_{2}^{-}\right)$and MPO from neutrophils. Cells were seeded into 24-well plates at a density of $5 \times 10^{5} \mathrm{cells} / \mathrm{mL}$, and three wells were allocated for each group.

A. The cells were treated with PMA $(200 \mathrm{ng} / \mathrm{mL})$, and (or) Dex $\left(10^{-6} \mathrm{~mol} / \mathrm{L}, 10^{-7} \mathrm{~mol} / \mathrm{L}, 10^{-8} \mathrm{~mol} / \mathrm{L}\right)$, and (or) Fas $(100 \mu \mathrm{mol} / \mathrm{L})[7]$. The reactions were terminated after 5,15 or $30 \mathrm{~min}$, and the $\mathrm{O}_{2}{ }^{-}$and MPO released from the neutrophils were measured according to the instructions provided with the kit.

B. Based on the results of the experiments described in 1.2.2 A, a Dex concentration of $10^{-6} \mathrm{~mol} / \mathrm{L}$ was used for the subsequent experiments. And RU-486 $\left(10^{-4} \mathrm{~mol} / \mathrm{L}\right)$ and CHX $\left(10^{-4} \mathrm{~mol} / \mathrm{L}\right)$ were used to evaluate whether they could prevent the effect of Dex. The reactions were terminated after $30 \mathrm{~min}$, and the released $\mathrm{O}_{2}$ - and MPO were measured as above.

Analysis of U937 and HUVEC cell adhesion. The cells were grouped as described in 1.2.2 B. HUVEC cells were seeded into 96 -well plates at a density of $4 \times 10^{3}$ cells per well and incubated for $12 \mathrm{~h}$. After the HUVEC cells had attached to the culture surface, $5 \times 10^{4} \mathrm{U} 937$ cells were added to each well. The cells were incubated for $30 \mathrm{~min}$ after stimulation, and after discarding the supernatant, the cells were gently washed 2 times with PBS. Ten microliters of CCK-8 solution was added to each well, followed by an incubation period of 1-2 $\mathrm{h}$. The optical density (OD) values, which indicate the extent of cell adhesion, were measured at a wavelength of $450 \mathrm{~nm}$ using an enzyme-linked immunosorbent assay (ELISA) plate reader.

Determination of ROCK1 activity. U937 cells were collected after the treatment. For each experimental group, ROCK1 protein was extracted from the U937 cells using a pull-down assay then aliquoted into Eppendorf (EP) tubes. Five microliters of anti-ROCK1 antibody (Santa Cruz, USA) was added to each tube, and the tubes were shaken at room temperature for 1-2 h. Twenty-five microliters of Protein A/G magnetic beads (Pierce, USA) was added to each tube, followed by thorough mixing of the solutions and incubation at room temperature for $1 \mathrm{~h}$. After incubation, the magnetic beads were collected and washed gently 2-3 times. ROCK1 protein was eluted from the beads and stored for the activity analysis [8]. ROCK1 activity was measured by ELISA according to the instruction manual for the ELISA kit used (Cyclex, Japan). The absorbance was measured using wavelengths of 450 and $540 \mathrm{~nm}\left(\mathrm{OD}_{450} / \mathrm{OD}_{540}\right)$.

Quantification of ROCK1 mRNA levels. Total RNA was isolated using the Trizol method, quantified using ultraviolet (UV) absorption and reverse transcribed using a reverse transcription kit (TaKaRa Bio Inc.). The resulting cDNA was quantified using a real-time fluorescence-based quantitative PCR kit (TaKaRa Bio Inc.). The following primers were used for the analysis: ROCK1 forward and reverse primers (5'-ATGAGTTTATTCCTACACTCTACCACTTTC-3' and 5'-TAACATGGCATCTTCGACACTCTAG-3', respectively) and glyceraldehyde 3-phosphate dehydrogenase (GAPDH) forward and reverse primers (5'-ACACCCACTCCTCCACCTTT-3' and 5'-TAGCCAAATTCGTTGTCATACC-3', respectively). The PCR reaction conditions were $95^{\circ} \mathrm{C}$ for $4 \mathrm{~min}$ and 40 cycles of $95^{\circ} \mathrm{C}$ for $30 \mathrm{~s}, 59^{\circ} \mathrm{C}$ for $15 \mathrm{~s}$ and $72^{\circ} \mathrm{C}$ for $15 \mathrm{~s}$ [9]. GAPDH was used as the internal reference.

Examination of RhoA activity. RhoA activity was measured using the RhoA activation assay kit (Neweast, USA). Equivalent amounts of total protein extracted from treated U937 cells were transferred to EP tubes, and the volume in each tube was adjusted to $1 \mathrm{~mL}$ using $1 \times$ lysis buffer. The protein extracts were mixed with $1 \mu \mathrm{L}$ of anti-active RhoA antibody and a $20 \mu \mathrm{L}$ volume of magnetic bead suspension then incubated at $4^{\circ} \mathrm{C}$ for $1 \mathrm{~h}$ with shaking. The magnetic beads were washed 3 times with $1 \times$ lysis buffer, collected and resuspended in $20 \mu \mathrm{L}$ of sample loading buffer. After boiling for $5 \mathrm{~min}$, the samples were centrifuged at 5000 $\mathrm{x} g$ for $10 \mathrm{~s}$. Western blot analysis was used to compare the levels of active RhoA protein in the treatment and control groups.

Examination of RhoA and ROCK1 protein expression. Total cellular protein was extracted and quantified. Thirty micrograms of total protein was mixed with $2 \times$ loading buffer, boiled, separated by sodium dodecyl sulfate polyacrylamide gel electrophoresis (SDS-PAGE) using a 7.5\% gel and transferred to a polyvinylidene difluoride (PVDF) membrane. The membrane was blocked at room temperature for $5 \mathrm{~h}$, incubated with anti-RhoA primary antibody (1:300 dilution, Neweast, USA) and anti-ROCK1 primary antibody (1:500 dilution, BD Biosciences, USA) at room temperature for $2 \mathrm{~h}$ and then mixed with goat anti-mouse/rabbit IgG secondary antibodies (1:5000 dilution, Sigma, USA). The proteins were visualized using an enhanced chemiluminescence (ECL) detection system and imaged. The intensity of each protein band was determined using Labworks 4.6 image analysis software, and $\beta$-actin was used as the internal reference [10]. 

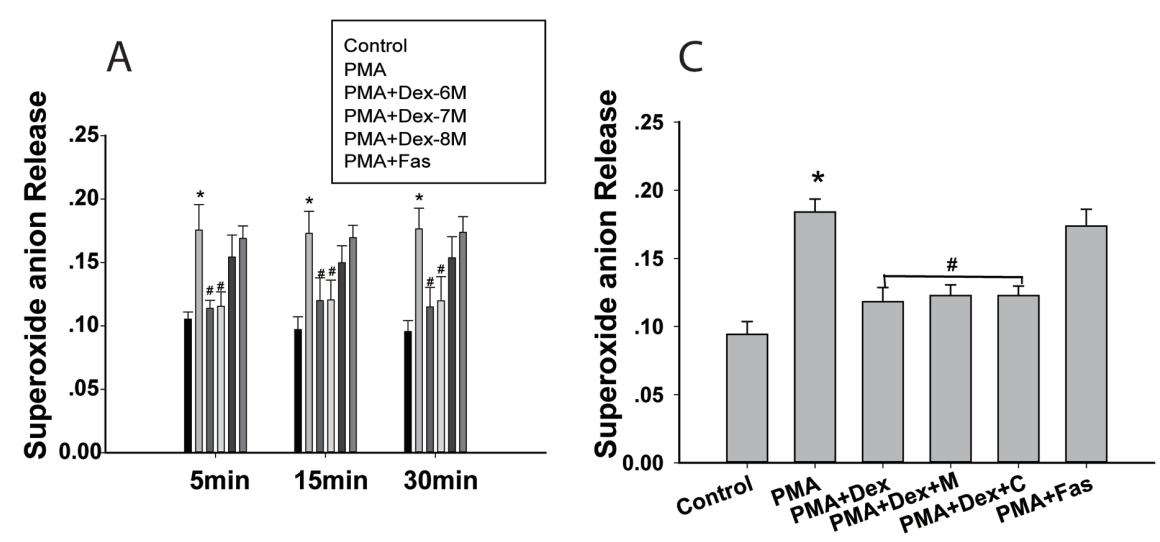

B

D
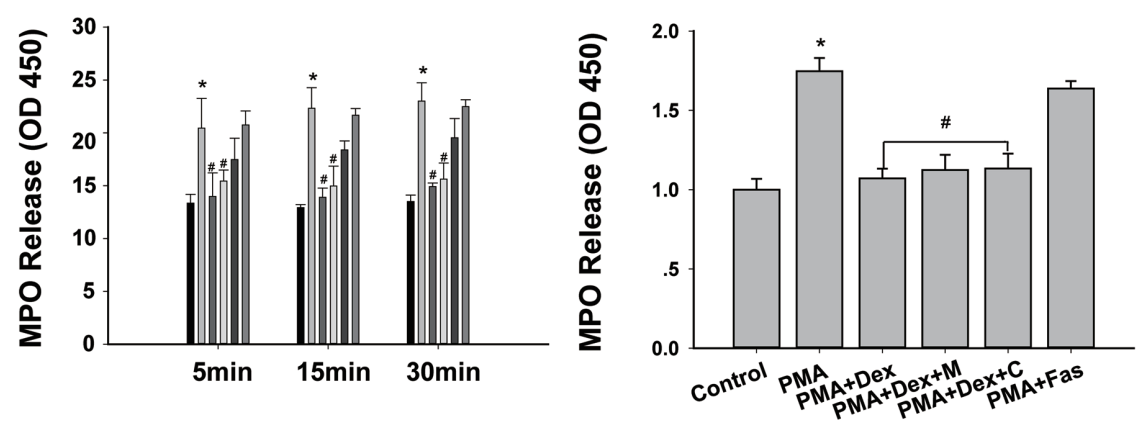

Fig. 1. Alterations of $\mathrm{O}_{2}^{-}(\mathrm{A})$ and MPO (C) in PMN. Cells were treated with Dex $\left(10-6 \mathrm{~mol} / \mathrm{L}, 10^{-7} \mathrm{~mol} / \mathrm{L}\right.$, $\left.10^{-8} \mathrm{~mol} / \mathrm{L}\right)$ and cells were examined in $5 \mathrm{~min}, 15 \mathrm{~min}$ and $30 \mathrm{~min}$ after PMA (200 ng/mL) stimulated. Furthemore, alterations of $\mathrm{O}_{2}^{-}(\mathrm{B})$ and MPO (D) in PMN. Cells were pretreated with $\operatorname{Dex}\left(10^{-6} \mathrm{~mol} / \mathrm{L}\right.$ ) and (or) M, C, Fas. Treatment with Dex decreased $\mathrm{O}_{2}^{-}$and MPO, andM and $\mathrm{C}$ did not block the Dex inhibition of PMA stimulation. *indicates $\mathrm{P}<0.05$, comparing the corresponding groups to the control group; \#indicates $\mathrm{P}<0.05$, comparing the corresponding groups to the PMA group. Data are expressed as the mean \pm S.E.M. One-way ANOVA and the Student-Newman-Keuls test were used, $n=4$ wells per group.

\section{Statistical analysis}

All of the experimental data are presented as the mean values \pm standard deviation. The graphics were obtained using Sigmaplot 11.0 software, and statistical analysis was performed using SPSS 17.0 software. Multiple group comparison was performed using univariate analysis of variance (ANOVA).

\section{Results}

The effects of PMA, Dex and Fas on the release of $\mathrm{O}_{2}$ and MPO from neutrophils

As shown in Fig. 1, compared to the control group, neutrophil stimulation with PMA $(200 \mathrm{ng} / \mathrm{mL})$ for 5, 15 and $30 \mathrm{~min}$ significantly increased the release of $\mathrm{O}_{2}{ }^{-}$and $\mathrm{MPO}(\mathrm{p}<0.05)$, indicating that the stimulation was effective.

Dex at $10^{-6} \mathrm{~mol} / \mathrm{L}$ and $10^{-7} \mathrm{~mol} / \mathrm{L}$ inhibited the stimulatory effect of PMA to varying degrees, and both concentrations significantly decreased neutrophil release of $\mathrm{O}_{2}{ }^{-}$and MPO $(\mathrm{p}<0.05)$. In contrast, significant inhibition of $\mathrm{O}_{2}^{-}$and MPO release by neutrophils was not observed with $10^{-8} \mathrm{~mol} / \mathrm{L}$ Dex or $100 \mu \mathrm{mol} / \mathrm{L} \mathrm{Fas,} \mathrm{which} \mathrm{specifically} \mathrm{inhibits} \mathrm{ROCK,} \mathrm{compared}$ to the control group ( $p>0.05$ ). The above effects of Dex were not time dependent, and the results of the experiments described in 1.2.2 B revealed that RU-486 or CHX did not block the $10^{-6} \mathrm{~mol} / \mathrm{L}$ Dex inhibition of PMA stimulation 
Fig. 2. Alterations of U937-HUVEC cell adhesion capacity. Cells were treated with Dex $\left(10^{-6} \mathrm{~mol} / \mathrm{L}\right)$ and (or) M, C, Fas and cells were examined in 30min after PMA (200 $\mathrm{ng} / \mathrm{mL}$ ) stimulated. The optical density (OD) values, which indicate the extent of cell adhesion. Treatment with Dex decreased cell adhesion, and $\mathrm{M}$ and $\mathrm{C}$ did not block the Dex inhibition of PMA stimulation. *indicates $\mathrm{P}<0.05$, comparing to the control group; \#indicates $\mathrm{P}<0.05$, comparing to the PMA group. Data are expressed as the mean \pm S.E.M. One-way ANOVA and the Student-Newman-Keuls test were used, $\mathrm{n}=4$ wells per group.
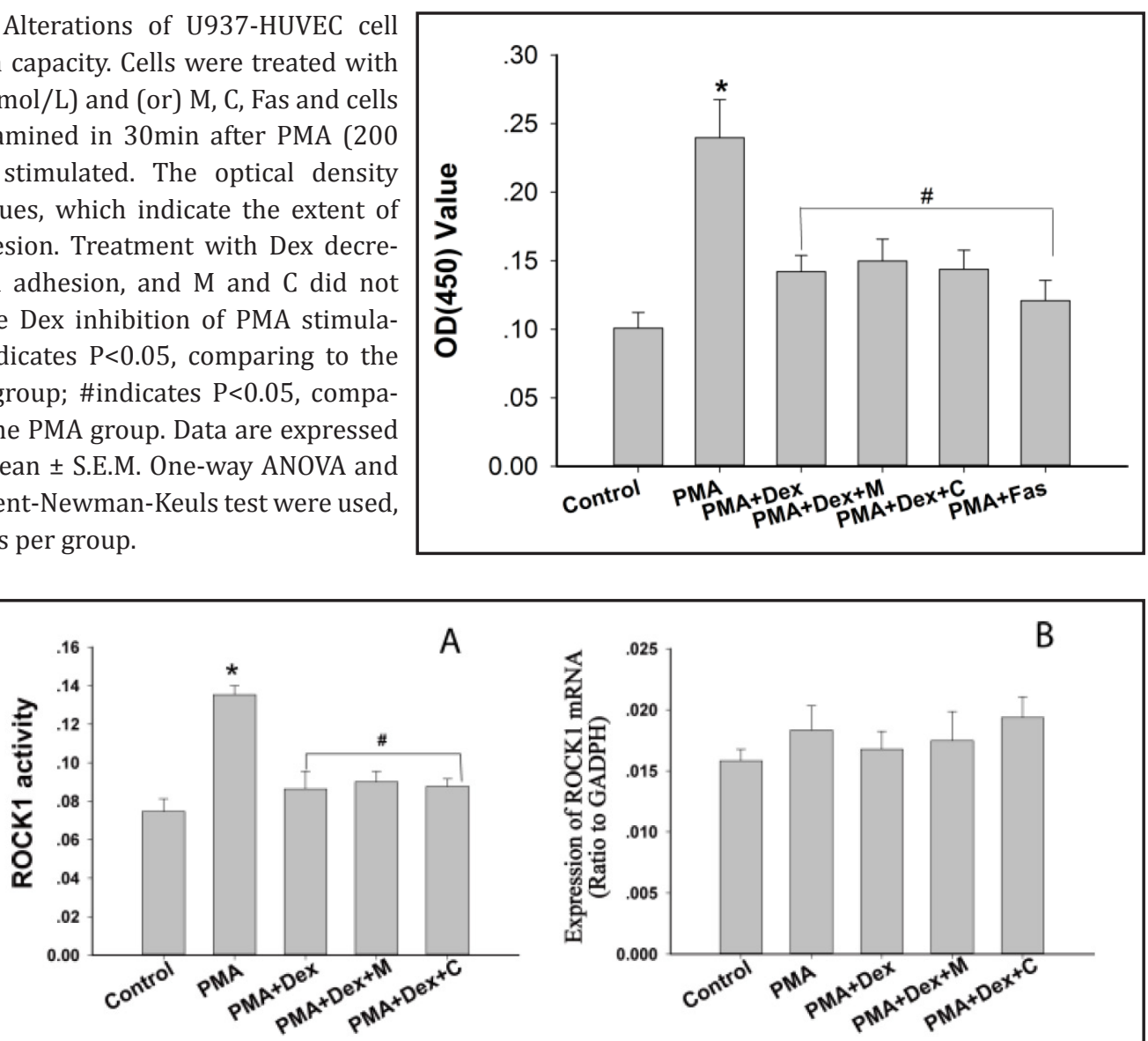

C

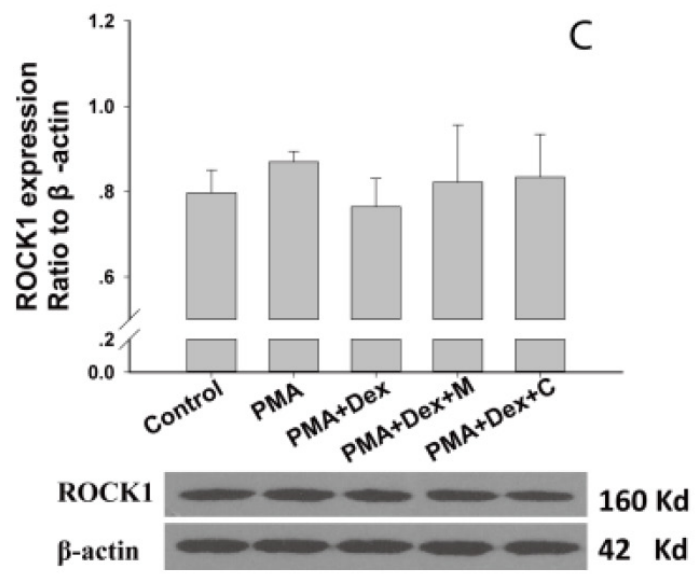

Fig. 3. Alterations of ROCK1 activity (A), mRNA expression (B) and protein expression (C) in U937 cell. Cells were treated with Dex $\left(10^{-6} \mathrm{~mol} / \mathrm{L}\right)$ and (or) M, C, Fas and cells were examined in $30 \mathrm{~min}$ after PMA (200 ng/ $\mathrm{mL}$ ) stimulated. *indicates $\mathrm{P}<0.05$, comparing the corresponding groups to the control group; \#indicates $\mathrm{P}<0.05$, comparing the corresponding groups to the PMA group. Data are expressed as the mean \pm S.E.M. One-way ANOVA and the Student-Newman-Keuls test were used, $n=4$ wells per group.

\section{U937 cell adhesion inhibited by both $10^{-6} \mathrm{~mol} / \mathrm{L}$ Dex and $10^{-6} \mathrm{~mol} / \mathrm{L} \mathrm{Fas}$}

As shown in Fig. 2, compared to the unstimulated group, stimulation with PMA significantly increased U937 cell adhesion $(p<0.01)$. Both Dex and Fas were found to significantly decrease the adhesion rate relative to the PMA stimulation group $(\mathrm{p}<0.01)$. In 


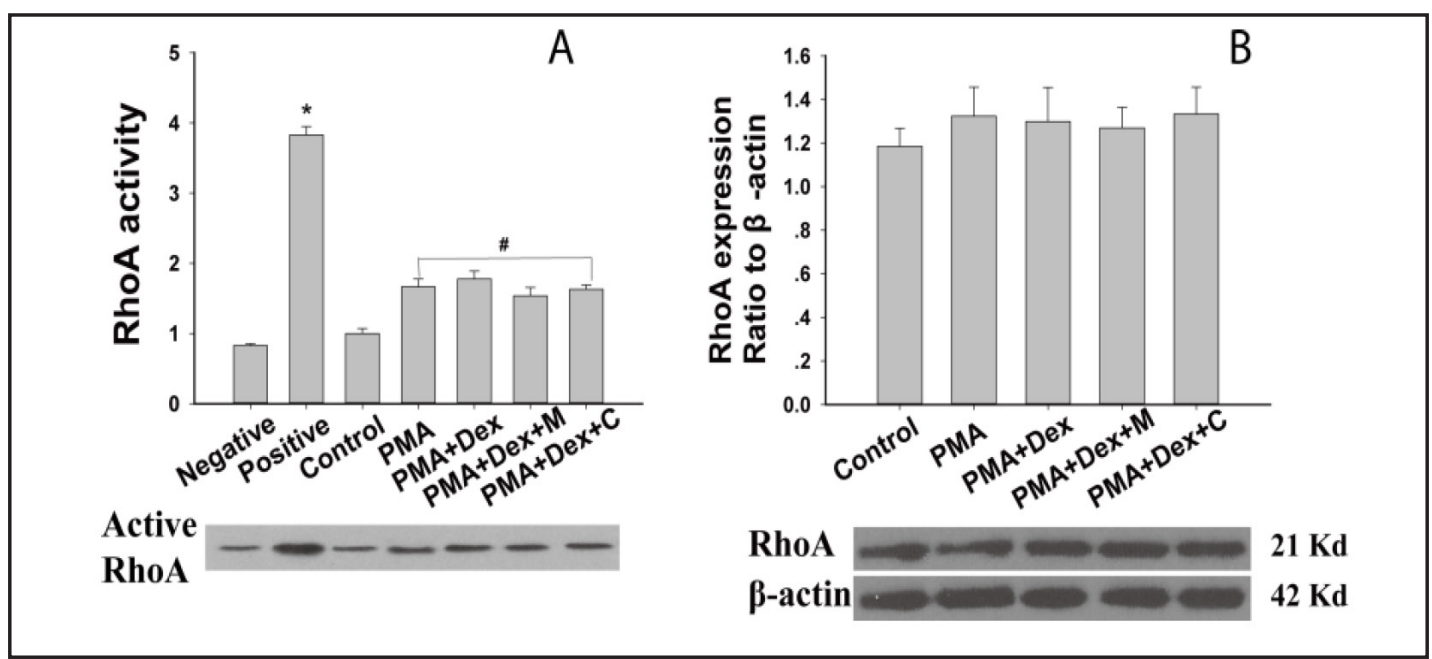

Fig. 4. Alterations of RhoA activity (A) and protein expression (B) in U937 cell. Cells were pretreated with Dex $\left(10^{-6} \mathrm{~mol} / \mathrm{L}\right)$ and (or) M, C, Fas and cells were examined in 30min after PMA (200 ng/mL) stimulated. *indicates $\mathrm{P}<0.05$, comparing the corresponding groups to the control group; \#indicates $\mathrm{P}<0.05$, comparing the corresponding groups to the PMA group. Data are expressed as the mean \pm S.E.M. One-way ANOVA and the Student-Newman-Keuls test were used, $n=4$ wells per group.

contrast, no significant differences were detected among the PMA+Dex+M, PMA+Dex+C and PMA+Dex groups $(\mathrm{p}>0.05)$.

Effects of Dex on the expression and activity levels of ROCK1 protein in U937 cells

As shown in Fig. 3A, stimulation of U937 cells with PMA significantly increased ROCK1 activity compared to the control group $(\mathrm{p}<0.05)$, while both Dex and Fas inhibited ROCK1 activity $(p<0.05)$. No significant differences were observed when comparing the PMA+Dex+M group or the $P M A+D e x+C$ group to the $P M A+D e x$ group ( $>0.05)$. In contrast to its effect on ROCK1 activity, Dex did not affect ROCK1 mRNA expression or protein levels in U937 cells (p>0.05, Fig. 3B and 3C).

Effects of Dex on the expression and activity levels of RhoA protein in U937 cells

Figure 4A shows that the activity of RhoA protein were significantly higher in the positive controls than in the negative controls $(\mathrm{p}<0.01)$, indicating good experimental quality. PMA significantly increased RhoA activity $(\mathrm{p}<0.05)$. No drastic differences in RhoA activity were detected among the PMA+Dex, PMA+Dex+M and PMA+Dex+C groups ( $p>0.05)$. As shown in Fig. $4 \mathrm{~B}$, no significantly differences in RhoA protein expression among the groups.

\section{Discussion}

The mechanisms underlying acute inflammation-induced body injury primarily involve damage to the vascular endothelial cells by cellular contents, such as $\mathrm{O}_{2}^{-}$and MPO released from neutrophils, and increased leukocyte adhesion to vascular endothelial and epithelial cells (cascade amplification), which is induced by the upregulated expression of various cytokines $[11,12]$. Therefore, inhibiting the release of cellular contents from neutrophils and reducing leukocyte adhesion to endothelial cells have become the key strategies for the treatment of acute inflammation.

Consistent with the findings of previous studies, our preliminary experiments showed that the rapid inhibitory effect of glucocorticoids on inflammation was dose dependent but not time dependent. High doses of Dex inhibited inflammatory responses effectively and 
rapidly (at 5, 15 and $30 \mathrm{~min}$ ). Moreover, $10^{-6} \mathrm{~mol} / \mathrm{L}$ Dex significantly inhibited the adhesion of U937 mononuclear cells to HUVEC, and neither the glucocorticoid receptor antagonist RU486 nor the protein synthesis inhibitor CHX suppressed this inhibition. Taken together, these results indicate that the inhibitory effects of Dex are mediated by non-genomic mechanisms as opposed to genomic mechanisms.

Previous studies have shown that downregulation of Rho kinase activity and inhibition of the Rho/ROCK signaling pathway reduce leukocyte adhesion in a variety of inflammatory responses [13]. In this context, ROCK has drawn increasing attention as a key inflammation regulator. For example, ROCK is known to play an important role in various inflammatory diseases, such as ALI, as well as in cardiovascular disease and ischemic brain damage [14, 15]. ROCK belongs to the serine/threonine protein kinase family and has two homologous isomeric forms, ROCK $\alpha /$ ROCK2 and ROCK $\beta /$ ROCK1. Whereas ROCK1 regulates adhesion and infiltration of the inflammatory cells, ROCK2 exhibits no such effects [16]. Accordingly, the ROCK1 subtype is recognized as a major factor involved in the adhesion of inflammatory cells, and we hypothesize that glucocorticoids reduce inflammatory responses through short-term non-genomic effects including inhibition of ROCK1 activity or expression.

The present findings show that the ROCK inhibitor Fas significantly decreased U937 cell adhesion in the short term but had no significant effects on the release of $\mathrm{O}_{2}^{-}$and MPO from neutrophils. These results suggest that ROCK1 is a key regulator of mononuclear cell adhesion but not of cellular content release from neutrophils. Dex significantly inhibited ROCK1 activity in the short term without affecting ROCK1 protein expression; moreover, the effects of Dex could not be reversed by RU-486 or CHX. Analyzing the expression and activity levels of ROCK1 protein showed significant decreases in ROCK1 activity in the Dex and Fas treatment groups compared to the PMA stimulation group. In contrast, no differences were observed in terms of ROCK1 protein abundance. Real-time PCR also revealed no changes in ROCK1 mRNA transcript levels following Dex treatment. Taken together, these results indicate that the effects of Dex did not involve the regulation of protein synthesis and that Dex inhibition of U937 cell adhesion and neutrophil content release was mediated through ROCK1-dependent non-genomic mechanisms and ROCK1-independent mechanisms, respectively.

As a key upstream effector of ROCK1, RhoA regulates ROCK1 activity and directly activates ROCK1 through interactions with the ROCK1 rho-binding domain (RBD) [17]. It remains unclear whether glucocorticoids affect ROCK1 activity through RhoA regulation. While ROCK1 activation is generally believed to be primarily dependent on RhoA [18], the present findings that Dex had no significant effects on RhoA protein expression or activity levels in the short term indicate that Dex inhibition of ROCK1 activity involved RhoAindependent signaling pathways. Nevertheless, the mechanisms by which Dex regulates ROCK1 activity remain to be elucidated. Rubenstein et al. have reported that Dex differentially regulates the activity and expression of ROCK1 and ROCK2 proteins [8] despite their high sequence similarity in the catalytic domain and the Rho-binding domain. We therefore hypothesize that the pleckstrin homology (PH) domain, for which ROCK1 and ROCK2 have $65 \%$ homology, is a key structure for differential regulation.

The PH domain is a functional protein domain approximately 120 amino acids in length. It is found in a wide range of signaling proteins and cytoskeleton-related proteins and mediates the interactions between signaling molecules in cellular signal transduction networks [19]. Although the functions of the PH domain are not yet fully understood, the following functions have been proposed: interaction with the $\beta / \gamma$ subunits of heterotrimeric guanosine nucleotide-binding proteins (G proteins) [20]; interaction with lipids and charged phospholipids, such as phosphatidylinositol $(4,5)$-bisphosphate $\left(\mathrm{PI}_{4,5} \mathrm{P}_{2}\right)[21]$; interaction with phosphorylated serine/threonine residues [22]; and membrane attachment through an unknown mechanism.

We hypothesize that external stimulation triggers the localization of ROCK1 from the cytoplasm to the cell membrane and that this process is mediated by the ROCK1 PH domain. Dex may potentially interact with this particular domain through membrane glucocorticoid 
receptors (mGCR) or through direct physical and chemical reactions with the cell membrane. Another possibility is that Dex interacts with the ROCK1 PH domain through the $\beta / \gamma$ subunits of $G$ proteins and thereby exerts its inhibitory effects [23]. The finding that Dex treatment did not affect the transcription or translation of the target proteins indicates that the effects of Dex involve non-genomic mechanisms; however, the molecular mechanisms underlying Dex activity remain to be elucidated.

In summary, the rapid inhibitory effect of Dex on inflammatory responses was found to involve the regulation of neutrophils and mononuclear cells through distinct mechanisms, presenting new ideas for understanding the mechanisms underlying the anti-inflammatory effects of glucocorticoids.

\section{Disclosure Statement}

The authors have not disclosed any potential conflicts of interest

\section{Acknowledgments}

This work was supported by grants from the National Natural Science Foundation of China (30470988) and the Ministry of Education Innovation Fund (IRT0712).

\section{Reference}

1 Li C, Yang P, Zhang Y, Sun Y, Wang W, Zou Z, Xing L, Chen Z, Tang C, Guo F, Deng J, Zhao Y, Yan Y, Tang J, Wang $\mathrm{X}$, Jiang C: Corticosteroid treatment ameliorates acute lung injury induced by 2009 swine origin influenza a (h1n1) virus in mice. PLoS One 2012;7:e44110.

-2 Bergquist M, Nurkkala M, Rylander C, Kristiansson E, Hedenstierna G, Lindholm C: Expression of the glucocorticoid receptor is decreased in experimental staphylococcus aureus sepsis. J Infect 2013;67:574583.

3 Altonsy MO, Sasse SK, Phang TL, Gerber AN: Context-dependent cooperation between nf-kb and the glucocorticoid receptor at a tnfaip3 enhancer: A mechanism to maintain negative feedback control of inflammation. J Biol Chem 2014;289:8231-8239.

4 Clark AR: Anti-inflammatory functions of glucocorticoid-induced genes. Mol Cell Endocrinol 2007;275:7997.

5 Slotta JE, Braun 00, Menger MD, Thorlacius H: Fasudil, a rho-kinase inhibitor, inhibits leukocyte adhesion in inflamed large blood vessels in vivo. Inflamm Res 2006;55:364-367.

6 Li XH, Peng J, Tan N, Wu WH, Li TT, Shi RZ, Li YJ: Involvement of asymmetric dimethylarginine and rho kinase in the vascular remodeling in monocrotaline-induced pulmonary hypertension. Vascul Pharmacol 2010;53:223-229.

7 Slotta JE, Braun OÖ, Menger MD, Thorlacius H: Fasudil, a rho-kinase inhibitor, inhibits leukocyte adhesion in inflamed large blood vessels in vivo. Inflamm Res 2006;55:364-367.

8 Rubenstein N, Callahan J, Lo D, Firestone G: Selective glucocorticoid control of rho kinase isoforms regulate cell-cell interactions. Biochem Biophys Res Commun 2007;354:603-607.

-9 Ho CC, Lai KC, Hsu SC, Kuo CL, Ma CY, Lin ML, Yang JS, Chung JG: Benzyl isothiocyanate (bitc) inhibits migration and invasion of human gastric cancer ags cells via suppressing erk signal pathways. Hum Exp Toxicol 2010;30:296-306.

10 Liu X, Li P, Liu P, Xiong R, Zhang E, Chen X, Gu D, Zhao Y, Wang Z, Zhou Y: The essential role for c-ski in mediating tgf-beta1-induced bi-directional effects on skin fibroblast proliferation through a feedback loop. Biochem J 2008;409:289-297. 
Liu et al.: Glucocorticoid Inhibts Inflammation via ROCK1

11 Gorudko IV, Grigorieva DV, Shamova EV, Kostevich VA, Sokolov AV, Mikhalchik EV, Cherenkevich SN, Arnhold J, Panasenko OM: Hypohalous acid-modified human serum albumin induces neutrophil nadph oxidase activation, degranulation, and shape change. Free Radic Biol Med 2014;68:326-334.

12 Lloyd MM, Grima MA, Rayner BS, Hadfield KA, Davies MJ, Hawkins CL: Comparative reactivity of the myeloperoxidase-derived oxidants hypochlorous acid and hypothiocyanous acid with human coronary artery endothelial cells. Free Radic Biol Med 2013;65:1352-1362.

13 Nübel T, Dippold W, Kleinert H, Kaina B, Fritz G: Lovastatin inhibits rho-regulated expression of e-selectin by tnf $\alpha$ and attenuates tumor cell adhesion. FASEB J 2004;18:140-142.

14 Rikitake Y, Oyama N, Wang CYC, Noma K, Satoh M, Kim HH, Liao JK: Decreased perivascular fibrosis but not cardiac hypertrophy in rock1+/- haploinsufficient mice. Circulation 2005;112:2959-2965.

15 Lu G, Hein TW, Kuo L: Rho kinase-mediated coronary arteriolar constriction to endothelin-1: Mechanistic implications for cardiac syndrome $\mathrm{x}$. Transl Biomed 2010;1.

16 Noma K, Rikitake Y, Oyama N, Yan G, Alcaide P, Liu P, Wang H, Ahl D, Sawada N, Okamoto R: Rock1 mediates leukocyte recruitment and neointima formation following vascular injury. J Clin Invest 2008;118:16321644.

17 Hall A: Rho gtpases and the control of cell behaviour. Biochem Soc Trans 2005;33:891-895.

18 Ishizaki T, Maekawa M, Fujisawa K, Okawa K, Iwamatsu A, Fujita A, Watanabe N, Saito Y, Kakizuka A, Morii $\mathrm{N}$ : The small gtp-binding protein rho binds to and activates a $160 \mathrm{kda}$ ser/thr protein kinase homologous to myotonic dystrophy kinase. EMBO J 1996;15:1885.

19 Haslam RJ, Koide HB, Hemmings BA: Pleckstrin domain homology. Nature 1993;363:309-310.

20 Wang D, Shaw R, Winkelmann J, Shaw G: Binding of ph domains of [beta]-adrenergic-receptor kinase and [beta]-spectrin to $\mathrm{wd} 40 /[$ beta]-transducin repeat containing regions of the [beta]-subunit of trimeric g-proteins. Biochem Biophys Res Commun 1994;203:29-35.

-21 Wang D, Shaw G: The association of the c-terminal region of I 1; ii spectrin to brain membranes is mediated by a ph domain, does not require membrane proteins, and coincides with a inositol-1, 4, 5 trisphosphate binding site. Biochem Biophys Res Commun 1995;217:608-615.

22 Yao L, Kawakami Y, Kawakami T: The pleckstrin homology domain of bruton tyrosine kinase interacts with protein kinase c. Proc Natl Acad Sci U S A 1994;91:9175.

23 Touhara K, Inglese J, Pitcher JA, Shaw G, Lefkowitz RJ: Binding of g protein beta gamma-subunits to pleckstrin homology domains. J Biol Chem 1994;269:10217-10220. 